\title{
Comportement longitudinal en flexion d'une conduite enterrée dans un remblai hétérogène
}

S.M. ELACHACHI

M. BENSAFI

M. HAMANE

D. NEDJAR

Département de Génie civil

Université des Sciences

et de la Technologie d'Oran

BP 1505 El menouar

Oran, Algérie

elachachi1@algeriecom.com

\section{BREYSSE}

CDGA

Université Bordeaux I avenue des Facultés 33405 Talence Cedex denys.breysse@cdga. $u$-bordeaux.fr
Un modèle simple permettant de modéliser l'interaction sol-conduite en tenant compte de la variabilité longitudinale du sol a été développé. Il est appliqué à l'analyse du comportement statique d'un tronçon de conduite d'assainissement. Une analyse probabiliste (méthode de Monte-Carlo) permettant de quantifier l'influence de la variabilité spatiale des caractéristiques géomécaniques du sol permet par une étude paramétrique non exhaustive de recenser et d'étudier les facteurs susceptibles d'influencer et de gouverner le comportement longitudinal d'un tronçon de canalisation. Le comportement du système est complexe, les interactions sol-structure étant gouvernées par trois types de rigídité : la rigidité du sol support, la rigidité des éléments de conduite (buses), la rigidité des joints entre buses voisines. Différentes analyses ont été menées afin de déterminer les variables dont les effets sont prépondérants. Une attention particulière a été portée sur l'influence de la longueur de corrélation des propriétés du sol et la rigidité des éléments de liaison.

Mots-clés : corrélation spatiale, interaction sol-structure, longueur de corrélation, réseaux enterrés, tassement différentiel, variabilité spatiale.
NDLR : Les discussions sur cet article sont acceptées jusqu'au 31 décembre 2004.
A simple model which describes the soil-pipe interaction and accounts for the longitudinal soil variation has been developed. It is used for the analysis of the static response of a section of a buried sewer. A probabilistic analysis (Monte-Carlo method) enabling to quantify the influence of spatial variability of the geomechanical characteristics of the soil makes possible to study the parameters which can influence and drive the Jongitudinal response of a section of sewer. The system response is complex, soil-structure interaction depending on three different stiffnesses: soil stiffness, pipe components stiffness and joints stiffness. Various analus is have been performed to identify the parameters whose influence is the larger. A specific attention has been devoted to the fluctuation scale of the soil properties and to the stiffness of joints.

Key words: buried sewers, differential settlements, fluctuation scale, soil-structure interaction, spatial correlation, spatial variability. 


\section{Introduction: variabilité spatiale, interaction sol-structure et désordres}

Les réseaux de conduites enterrées (réseaux d'assainissement, conduites d'eau potable ou de gaz) subissent des désordres que l'on peut relier, pour une grande part, à des mouvements de sol selon la direction longitudinale des tronçons de conduites qui provoquent des tassements différentiels. Les conséquences de ces tassements varient selon la nature des matériaux constitutifs des conduites. Ils sont susceptibles d'entrainer l'ouverture des joints ou la fissuration des conduites et par conséquent des fuites de liquide qui, à leur tour, en modifiant les caractéristiques du milieu environnant, induisent des tassements supplémentaires.

Dans les calculs usuels de dimensionnement (MELT, 1992), le comportement n'est modélisé que dans la section droite (comportement transversal), l'homogénéité aussi bien du remblai que du lit de pose étant des hypothèses de travail incontournables, alors que la dimension longitudinale (interaction solconduite) et la variabilité des caractéristiques du sol devraient intervenir dans ladite conception. Cette démarche simplifiée s'explique par la complexité de l'interaction sol-conduite, la prédiction correcte du comportement du système demandant à la fois une acquisition de données géotechniques pertinentes et le développement d'un modèle mécanique adapté.

De nombreuses études (Baecher et al., 1981; Liu et al., 1987; Bucher et al., 1988; Der Kiureghian et al., 1988; Grundmann et al., 1993; Carmeliet et al., 1994; Cheng et al., 1994; Zhang et al.,1995; Fenton et al., 1995) ont été menées sur l'effet de l'interaction sol-structure sous un chargement statique où sont combinés l'efficacité de la méthode classique des éléments finis et les possibilités d'une approche probabiliste et une modélisation stochastique. Un nombre cependant limité d'études (Tíng et al., 1984; Kouraoaka, 1993; Benmansour et al., 1997; Bensafi et al., 2002) a été conduit sur l'effet de l'interaction sol-structure considérant les conduites enterrées.

Afin que les réseaux d'assainissement puissent assumer pleinement leur fonction et ce de façon pérenne, il est indispensable de maitriser les effets de la variabilité longitudinale du sol de remblai (Breysse et Boissier, 2002). Cette maitrise peut s'effectuer:

- au moment de la phase de réalisation, en «homogénéisant m les caractéristiques du sol par le choix du matériau de remblai (granulométrie, teneur en eau et état de densité), par le contróle de la compacité (essai Proctor) et par une mise en place soigneuse des conduites:

- ou bien en amont lors de la phase de conception. en adoptant une démarche où sont intégrés les « ingrédients ») suffisants pour pouvoir tenir compte de la variabilité du sol, d'une part, et des incertitudes liées à la méconnaissance de ses caractéristiques, d'autre part.

\section{2}

\section{Application de la méthode de Monte-Carlo}

L'approche adoptée consiste à combiner la méthode classique des éléments finis avec les possibilités d'une modélisation stochastique (probabiliste). Les méthodes stochastiques sont composées principalement de deux familles: les méthodes de perturbation et la méthode de Monte-Carlo. Chaque simulation regroupe trois étapes:

a) une discrétisation du champ aléatoire, censé reproduire la variation spatiale des propriétés du sol de remblai:

b) une analyse par éléments finis pour estimer, via un calcul déterministe, la réponse du système;

c) une analyse statistique de la réponse de la structure, qui permet d'estimer par exemple les risques de dépassement de telle ou telle valeur critique (de moment, de contrainte, de déplacement...).

La méthode de Monte-Carlo consiste pour une même confiquration géométrico-matérielle, à générer une série de réalisations particulières des propriétés de la structure (tirages au sort, respectant les distributions statistiques données a priori). Une solution déterministe est ensuite obtenue à partir de la méthode des éléments finis P2 pour chaque réalisation, la procédure étant répétée jusqu'ả ce que les résultats puissent être considérés comme statistiquement représentatifs (stabilité de la réponse) avec la précision désirée. Une analyse statistique de l'ensemble des réponses est menée à l'issue des simulations et permet de déduire des informations utiles à la conception du système.

\section{3}

\section{Définition du système (réseau de conduites-sol-chargement)}

Un réseau est constitué de plusieurs tronçons généralement délimités (Fig. 1) par des regards. Chaque tronçon est un assemblage de buses de lonqueur normalisée (de 1 à $6 \mathrm{~m}$ ) reliées entre elles par des joints de liaison (d'étanchéité). Il est généralement enterré et repose directement sur le sol. Différents modèles ont été développés pour tenir compte de l'interaction sol-structure. On citera le modèle classique de Winkler (1867), et les améliorations successives qu'en ont proposé Filonenko-Borodich (1940), Pasternak (1954), Hetenyi (1950), Kerr $(1964,1965)$ et Horvarth $(1983,1993)$. Dans cette étude et malgré ses limites, nous avons choisi d'utiliser le modèle de Winkler, pour deux raisons:

- sa simplicité d'utilisation et de mise en ceuvre;

- sa capacité à prendre en compte de manière indirecte la variabilité des caractéristiques du sol.
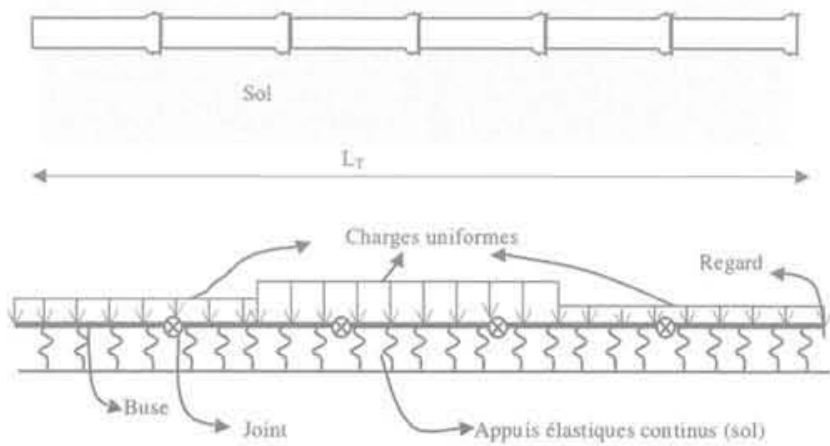

FIG.1 Modélisation d'un tronçon de conduites. Pipe sections modeling. 


\section{L'action exercée (chargement)}

En pratique, elle résulte du poids du remblai surplombant la conduite, de la charge d'exploitation (due par exemple aux véhicules), de la pression hydrostatique due à la nappe phréatique, du poids propre de la conduite et également des actions internes du fluide véhiculé. Dans les exemples traités, nous considérerons que le chargement appliqué est constitué d'une action verticale répartie et uniforme, d'intensité constante q $(\mathrm{N} / \mathrm{ml})$, Il est cependant possible, avec le même modèle, de simuler l'effet de sollicitations plus complexes, par exemple d'origine sismique, donc rapidement variables dans le temps (Bensafi et al., 2002; Nedjar et al., 2002).

\section{2}

\section{La conduite}

Les matériaux utilisés pour la réalisation d'une conduite sont divers: pierre (grès), béton ou liants cimentaires, fonte ou acier, PVC... Leur diamètre varie en pratique de 0,15 à $1,5 \mathrm{~m}$ pour les conduites non visitables et de 1,5 à $3 \mathrm{~m}$ pour les conduites dites visitables (dont on peut faire l'inspection). L'étanchéité au niveau de la jonction des conduites est assurée par des joints de raccord de type plastique, de type mortier de ciment ou plus fréquemment de type élastomère. La rigidité de ces joints est aussi variable que les technologies et géométries employées : elle peut être très faible (joints souples) ou très élevée (joints soudés).

\section{3}

\section{Le coefficient de réaction du sol}

Le sol oppose aux éléments constitutifs d'une conduite (buses) une réaction continue $\mathrm{R}(\mathrm{x})$, de dimension [F/L], donnée par l'expression (1):

$$
R(x)=S_{s}(x) \cdot D_{\text {ext }}
$$

où $S_{s}(x)$ est la contrainte (sous la buse) et $D_{\text {exx }}$ le diamètre extérieur de la buse.

La contrainte s'exprime selon le modèle de Winkler sous la forme:

$$
\mathrm{S}_{5}(\mathrm{x})=\mathrm{k} \cdot \mathrm{W}(\mathrm{x})
$$

où $k$ est le coefficient de réaction du sol (ou constante de proportionnalité de Winkler) de dimension [F/L'3 et $\mathrm{w}(\mathrm{x})$ la flèche de la conduite (et donc le tassement du sol).
Selon la théorie d'élasticité, la réponse d'un corps élastique à un chargement devrait être caractérisée par au moins deux paramètres, tels que le module d'élasticité et le coefficient de Poisson. Le sol est un matériau non élastique et non homogène, et une caractérisation précise de son comportement mécanique exige habituellement plus de deux paramètres.

L'utilisation d'un seul paramètre tel que le module de la réaction du sol $\mathrm{k}$ pourrait donc apparaître excessivement simplificatrice. Cependant, une telle approche semble cohérente compte tenu de la variabilité et des incertitudes liées à la caractérisation d'un sol. Les aspects non linéaires du comportement peuvent être reproduits simplement: il suffit d'adopter des lois $k(t)$ ou $\mathrm{k}(\mathrm{w})$ selon que l'on souhaite décrire une dégradation progressive des propriétés ou une non-linéarité matérielle... la difficulté est d'identifier de telles lois. Si le modèle de Winkler est unidimensionnel, et donc inapte en principe à reproduire les effets des cisaillements dans le sol, les corrélations spatiales que nous introduirons au $\$ 4$ conduisent à assurer, de fait, une cohérence des déplacements en des points voisins telle qu'elle existe dans un milieu continu.

Notons que le module de réaction k n'est pas simplement un paramètre de sol: il est également affecté par la rigidité de la conduite. Il dépend par conséquent de plusieurs facteurs, tels que la longueur et la largeur de la conduite, la profondeur de pose, le type de matériau utilisé et du type du lit de pose. Il est d'autant plus élevé que le sol est rigide et la conduite souple.

La valeur de $k$ n'est accessible que par des méthodes semi-empiriques (Vesic, 1961; Biot, 1937; Vlassov, 1956; Meyerhof, Kloppel selon Okeagu et al., 1984; Matsubara, 2000). Chacun des auteurs est parvenu à une expression propre (les formules correspondantes sont données en Annexe 1), ce qui souligne l'incertitude attachée au modèle de réaction (les mêmes questions se posent pour la rigidité du sol derrière un écran souple ou sous une semelle de fondation). Pour un même jeu de valeurs (propriétés mécaniques et géométriques du sol et de la conduite, données au tableau I), le tableau II illustre l'étendue de la gamme de valeurs obtenues pour $\mathrm{k}$ selon la formule empirique employée.

Ajoutons que l'expression de Matsubara requiert la connaissance d'un paramètre supplémentaire, $\lambda$, défini comme le rapport entre le rayon de la conduite et la distance (rayon) par rapport au point du sol où le déplacement est considéré comme nul. L'ordre de grandeur de ce paramètre étant non défini, il a été pris égal à 10. On peut noter que $\mathrm{k}$ varie du simple au triple selon les auteurs, et quasiment du simple au double si l'on néglige les valeurs extrêmes (Vesic et Kloppel).

TABLEAUI Valeurs de référence des caractéristiques de la conduite, Pipe characteristics.

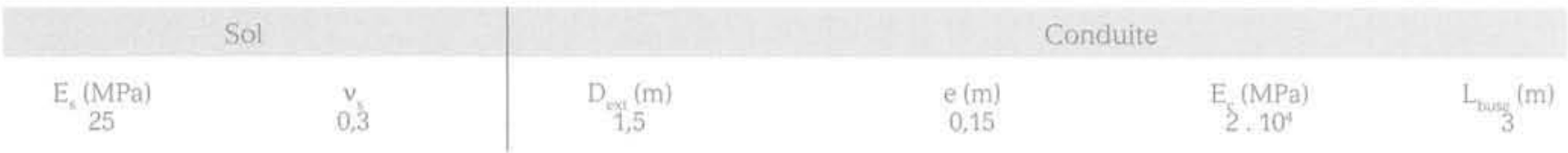

TABLEAU II Valeurs du coefficient de réaction du sol k.

Coefficient of subgrade reaction values.

\begin{tabular}{lccccccc} 
& Vesic & Meyerhof & Kloppel & Matsubara & Biot & Vlassov & Selvadurai \\
\hline$k\left(\mathrm{MN} / \mathrm{m}^{3}\right)$ & 9,16 & 18,32 & 25,64 & 17,49 & 12,39 & 22,43 & 11,90 \\
\hline
\end{tabular}


Cette relative imprécision sur la valeur de $\mathrm{k}$ peut être comparẻe à la gamme attendue de variation de $\mathrm{k}$ pour des configurations géométrico-matérielles "balayant " l'espace de variations des paramètres considérés. Ainsi, en considérant des valeurs minimales et maximales raisonnables pour le contraste de raideur entre le sol et la conduite (Tableau III), on obtient une variation relative de $\mathrm{k}$ de 1 à 20 (soit de $5-15$ $\mathrm{MN} / \mathrm{m}^{3}$ à $100-250 \mathrm{MN} / \mathrm{m}^{3}$ ).

Dans un second temps, nous avons analysé les rôles respectifs des propriétés du sol et de la conduite, les valeurs non précisées des paramètres étant prises égales à celles de la configuration de référence:

- quand le module d'Young du sol passe de 10 à $50 \mathrm{MPa}, \mathrm{k}$ passe de $5-15 \mathrm{MN} / \mathrm{m}^{3}$ à $30-80 \mathrm{MN} / \mathrm{m}^{3}$.

- quand la conduite passe d'un diamètre de $0,20 \mathrm{~m}$ à $2 \mathrm{~m}$, k passe de $70-200 \mathrm{MN} / \mathrm{m}^{3}$ à $7-20 \mathrm{MN} / \mathrm{m}^{3}$.

La figure 2 présente, pour les différents auteurs, l'évolution du coefficient de réaction du sol $\mathrm{k}$ en fonction du module d'Young E. (Fig. 2a), et de celui de la conduite $E_{\text {f }}$ (Fig. 2b), chacun des paramètres fixes étant égal à la valeur donnée dans le tableau I. Les gammes de variation relevées (de 1 à 6 dans le premier cas, de 10 à 1 dans le second, de 1 à 20 en considérant des configurations extrêmes) illustrent les valeurs de $\mathrm{k}$ qu'il est raisonnable de considérer dans les simulations numériques et relativisent les écarts (de l'ordre de 1 à 3) observés sur les modèles du module de réaction. Par la suite, les études de sensibilité seront faites en tenant compte des ordres de grandeur des variations attendues de $\mathrm{k}$.

Retenons de ces constatations que, si des variations spatiales de k peuvent être interprétées comme résultant de la variabilité spatiale des propriétés du sol, il ne faudra pas s'attacher trop exclusivement aux valeurs de ce paramètre, une incertitude de l'ordre de $100 \%$ pouvant résulter du simple choix du modèle qui le définit.
De mème, en fixant le modèle, on pourra étudier l'influence de tel ou tel paramètre (par son poids sur la valeur de k), mais il sera préférable d'en rester à des considérations comparatives. En pratique, la valeur de $\mathrm{k}$ (et donc la distribution statistique de cette variable) ne peut pas être mesurée directement, puisque c'est un paramètre qui combine les propriétés du sol et de la conduite: toute sollicitation du sol via un dispositif quelconque (ne serait-ce qu' une plaque) fait intervenir à la fois les propriétés du sol et du dispositif utilisé. La procédure consiste donc à identifier sur le site la distribution statistique de la raideur $\mathrm{E}_{\text {(soit directement par }}$ des méthodes géophysiques, soit via des corrélations, par exemple avec un pénétromètre) puis à calibrer le modèle reliant $E_{\text {s }}$ et les caractéristiques géométriques au coefficient de réaction du sol $\mathrm{k}$.

\section{4}

\section{Prise en compte de la variabilité du coefficient de réaction du sol $\mathrm{k}$}

La variabilité et l'incertitude relatives aux caractéristiques du sol et par conséquent au coefficient de réaction du sol ont trois sources:

- l'hétérogénéité spatiale qui résulte du processus de formation et d'agrégation du sol, qu'il soit naturel ou qu'il s'agisse d'un milieu anthropique (Jaksa, 1995); - l'incertitude liée à l'imprécision des mesures (différences éventuelles entre les valeurs mesurées et les vraies valeurs, inconnues, de la caractéristique considérée):

- l'incertitude de modèle liée, comme cela a été montré $§ 3.3$, au fait que le module de réaction résulte de modèles semi-empiriques.

TABLEAU iII Domaine " balayé " par les différents paramètres. Field covered by the various parameters.

\begin{tabular}{lccccc}
\multicolumn{1}{c}{ Valeurs } & $\mathrm{D}_{\text {ed }}(\mathrm{m})$ & $\mathrm{e}(\mathrm{m})$ & $\mathrm{E}_{s}(\mathrm{MPa})$ & $\mathrm{E}_{\mathrm{s}}(\mathrm{MPa})$ & $v_{\mathrm{x}}$ \\
Conduite souple (PVC), sol raide & 0,3 & 0,015 & 2500 & 50 & 0,3 \\
Conduite raide (béton armé), sol souple & 2 & 0,15 & 30000 & 20 & 0,3
\end{tabular}
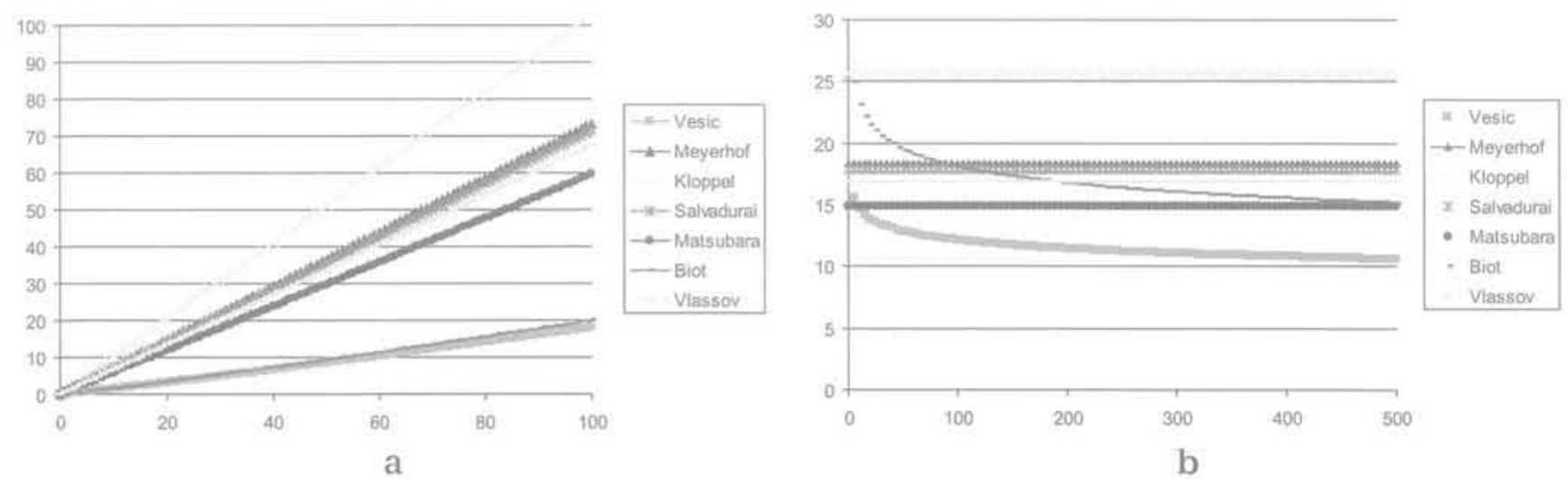

FiG. 2 Module de réaction $\mathrm{k}\left(\mathrm{MN} / \mathrm{m}^{3}\right)$ en fonction du : a) module de Young du sol $\mathrm{E}_{\mathrm{t}}(\mathrm{MPa})$; b) module de Young de la conduite E(MPa).

Modulus of subgrade reaction $\mathrm{k}(\mathrm{MN} / \mathrm{m} 3)$ function of : a) Soil Young modulus $\mathrm{E}_{\mathrm{s}}(\mathrm{MPa})$; b) Pipe Young modulus $\mathrm{E}_{\text {. }}(\mathrm{MPa})$. 
Le rôle de la variabilité longitudinale du remblai entourant la conduite nous paraissant essentiel, nous avons choisi de la modéliser en utilisant la théorie de la moyenne locale d'un champ aléatoire développée par VanMarcke (1983).

Le champ aléatoire du coefficient de réaction du sol $k(x)$ est défini par trois grandeurs: sa valeur moyenne $m$, sa variance $\sigma^{2}$ et son échelle (ou longueur) de corrélation 1 (liée à une fonction d'auto-corrélation $p(\tau)$, où $\tau$ désigne la distance entre deux points du sol et qui traduit la structure spatiale de corrélation des propriétés: $p(\tau)$ diffère selon que les propriétés varient plus ou moins rapidement en s'écartant d'un point considéré). Cette longueur de corrélation (longueur à partir de laquelle les propriétés ne sont plus corrélées) dépend de la caractéristique considérée du sol (module, porosité, teneur en eau...) et de la direction (horizontale ou verticale).

Le sol est discrétisé dans la direction longitudinale en considérant des zones où les propriétés sont uniformes (Fig. 3a). La valeur du champ aléatoire dans chaque zone est estimée par la moyenne du champ spatial $\mathrm{k}(\mathrm{x})$ de ladite zone. La moyenne locale ainsi que la variance dans la zone i de longueur D s'exprime:

$$
\begin{gathered}
E\left[k\left(D_{i}\right)\right]=m \\
\operatorname{var}\left[k\left(D_{i}\right)\right]=\sigma^{2} \gamma\left(D_{i}\right)
\end{gathered}
$$

a

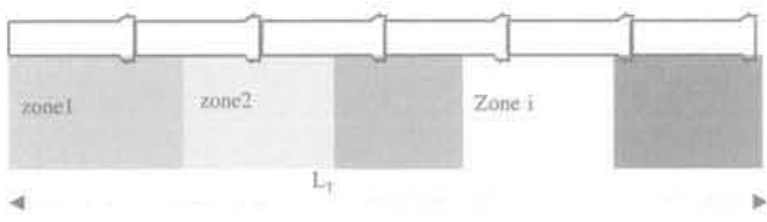

b
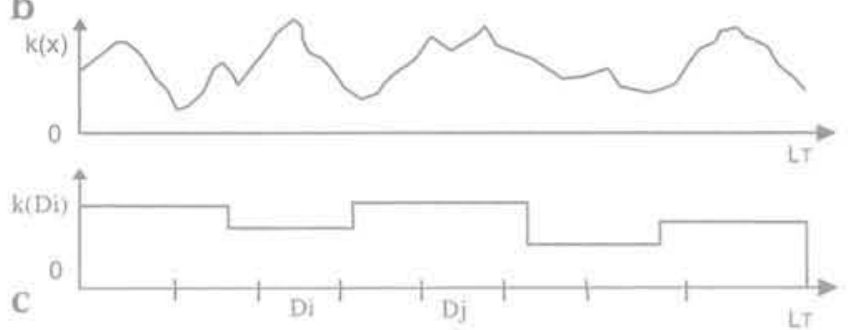

FIG. 3 Discrétisation du champ aléatoire $\mathrm{k}(\mathrm{x})$ et subdivision du sol en plusieurs zones. Discretization of random field $k(x)$.
La valeur moyenne est considérée constante pour tout le champ, quelle que soit la position du point $\mathrm{x}$. L'expression (3b) montre que la variance locale de $k(D)$ dépend de la longueur $\mathrm{D}$ de la zone $\mathrm{i}$, en suivant une loi qualifiée de «réduction de variance », $\gamma\left(D_{i}\right)$ est la fonction de variance du champ $k(x)$, c'est une mesure de la réduction de la variance due à la ("moyennisation » du processus aléatoire selon la longueur de la conduite. Elle est reliée à la fonction de corrélation par:

$$
\gamma\left(D_{i}\right)=\frac{2}{D_{i}} \int_{0}^{D_{i}}\left(1-\frac{x}{D_{i}}\right) \rho(x) d x
$$

En considérant la fonction de corrélation la plus simple, à savoir une fonction linéaire, et à partir de l'expression (4), on exprime la fonction variance en fonction de l'échelle de corrélation $1_{\text {ec }}$

$$
\begin{aligned}
& \gamma\left(D_{i}\right)=1-\frac{D_{i}}{3 l_{\text {ec }}} \quad \text { si } \quad D_{i} \leq I_{\mathrm{cs}} \\
& \gamma\left(D_{i}\right)=\frac{I_{c c}}{D_{i}}\left(1-\frac{I_{c c}}{3 D_{i}}\right) \quad \text { si } D_{i} \geq I_{\text {is }}
\end{aligned}
$$

Le passage à l'ensemble de la conduite passe par l'expression de la matrice de covariance (dont chaque terme $C_{i j}=\operatorname{Cov}[k(D), k(D)]$ correspond à la corrélation entre deux zones D et D), qui résulte d'une combinaison linéaire des fonctions variance (6).

$$
\operatorname{Cov}\left[k\left(D_{i}\right), k\left(D_{i}\right)\right]=\frac{\sigma^{2}}{2}\left\{(t-1)^{2} \gamma[(t-1) D]-2 t^{2} \gamma[t D]+(t+1)^{2} \gamma[(t+1) D]\right\}
$$

On utilise l'algorithme de Fenton et VanMarcke (1990) pour générer des variables aléatoires de distribution normale de moyenne nulle avec la structure de corrélation spatiale souhaitée. On passe dans un second temps à la variable $k$ respectant la distribution statistique visée.

La figure 4 compare les matrices de covariance théorique et simulée [C $]$ pour un sol subdivisé en 25 zones d'égale longueur. Les termes diagonaux ( $i=j)$ sont égaux à l'unité et la corrélation décroit quand la distance entre i et j augmente. L'identité des valeurs théoriques et des valeurs simulées valide le processus de simulation du champ aléatoire $\mathrm{k}(\mathrm{x})$.
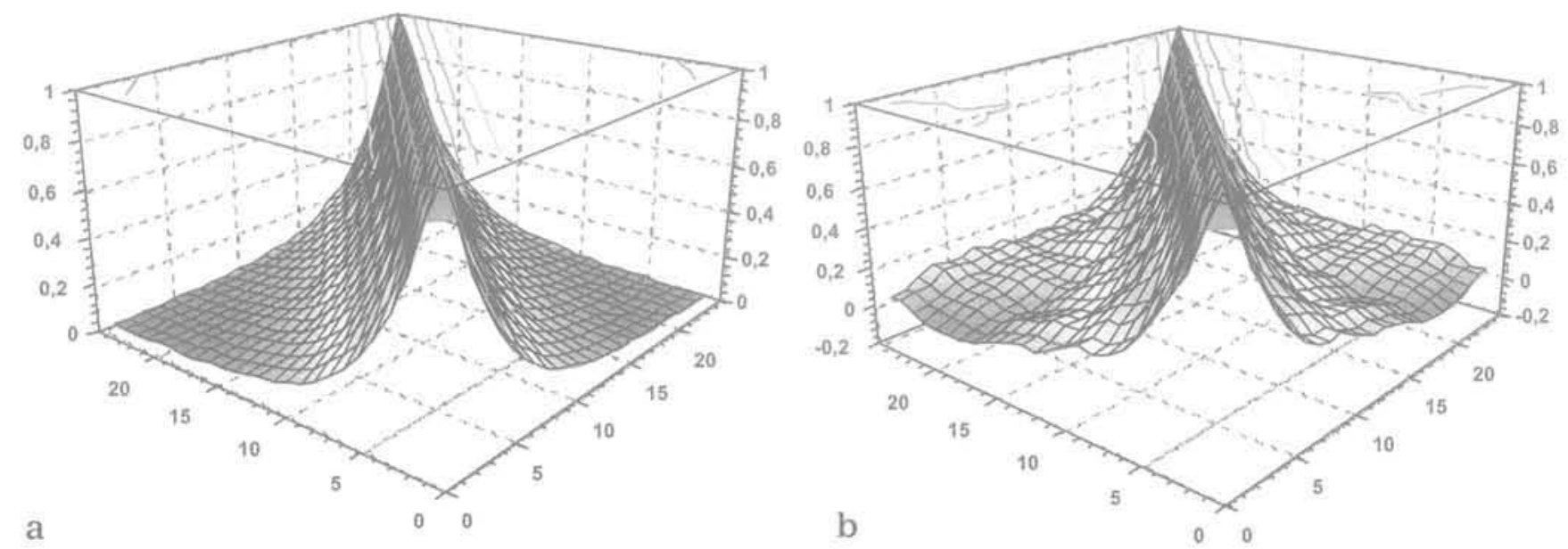

FIG.4 Matrices de covariance : a) théorique; b) simulée.

Matrices of covariance: a) theoretical; b) simulated. 


\section{Méthode des éléments finis}

Un programme adoptant la méthode des éléments finis classique a été développé. Les éléments utilisés sont de type poutre P2. Le tronçon est subdivisé en un ensemble de poutres interconnectées. Chaque poutre représente soit un tronçon de buse, soit une buse entière.

\section{1}

\section{Matrice de rigidité du système sol-conduite}

L'énergie de déformation $\psi$ du système sol-conduite est égale à la combinaison des énergies de déformation de la conduite $\psi_{1}$ et du sol $\psi_{2}$ :

$$
\psi=\psi_{1}+\psi_{2}
$$

dont les expressions sont formulées comme suit (éq. 8):

$$
\begin{aligned}
& \Psi_{1}=\frac{1}{2} \int_{0}^{l e} E_{c} I\left(\frac{\partial^{2} w(x)}{\partial x^{2}}\right)^{2} d x \\
& \Psi_{2}=\frac{1}{2} \int_{0}^{l e} k D_{\text {ext }} w(x) \cdot w(x) d x
\end{aligned}
$$

où E et I représentent respectivement le module d'élasticité et le moment d'inertie de la conduite et $l_{\text {e }}$ la longueur de l'élément fini.

En considérant une fonction de déplacement de type polynomial du troisième degré, on a :

$$
w(x)=[N]^{t}\{\delta\}
$$

de telle sorte que les inconnues sont les composantes du vecteur déplacement $\delta^{e}$ d'un élément et [N] la matrice composée des fonctions d'interpolations. Ce qui nous donne l'expression finale de $\psi_{1}$ et de $\psi_{2}$ :

$$
\begin{aligned}
& \Psi_{1}=\frac{1}{2}\left\{\delta^{e}\right\}^{t}\left(E_{I} \int_{0}^{l e}\left(\frac{\partial^{2} \mathrm{~N}}{\partial \mathrm{x}^{2}}\right)\left(\frac{\partial^{2} \mathrm{~N}}{\partial \mathrm{x}^{2}}\right) \mathrm{dx}\right)\left\{\delta^{e}\right\} \\
& \Psi_{2}=\frac{1}{2}\left\{\delta^{e}\right\}^{t}\left(k \mathrm{D}_{\mathrm{e} x+1} \int_{0}^{\mathrm{e}}\{\mathrm{N}\}\{\mathrm{N}\}^{t} \mathrm{dx}\right)\left\{\delta^{\mathrm{c}\}}\right.
\end{aligned}
$$

Sachant que l'énergie de déformation totale s'exprime par la relation (11) suivante:

$$
\Psi=\frac{1}{2}\left\{\delta^{e}\right\}^{t}\left[K^{e}\right]\left\{\delta^{e}\right\}
$$

où $\mathrm{K}^{\mathrm{e}}$ représente la matrice de rigidité de l'élément et en identifiant avec (10) on obtient:

$$
K^{e}=E I \int_{0}^{l e}\left(\frac{\partial^{2} N}{\partial x^{2}}\right)\left(\frac{\partial^{2} N}{\partial x^{2}}\right)^{t} d x+K D_{e x t} \int_{0}^{l e}\{N\}\{N\}^{t} d x
$$

oubien:

$$
\mathrm{K}_{\mathrm{p}}^{\mathrm{c}}=\mathrm{K}_{\mathrm{p}}^{\mathrm{e}}+\mathrm{K}_{\mathrm{s}}^{\mathrm{e}}
$$

On distingue deux termes dans les expressions (12) ou (13) ; au premier terme correspondant à la matrice de rigidité classique d'un élément poutre P2, s'ajoute un second terme qui traduit l'interaction sol-conduite. Le développement de l'expression (12) donne l'expression (14):

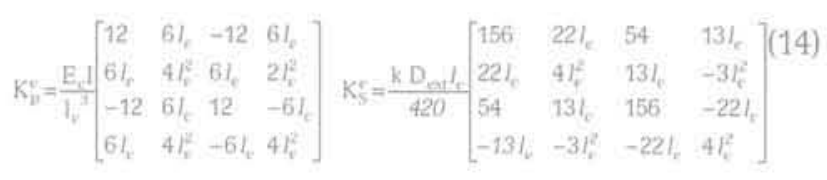

\section{2}

\section{Prise en compte du joint de liaison entre buses}

Pour modéliser la rotation différentielle possible des extrémités de deux buses adjacentes, chaque noud peut avoir trois degrés de liberté: une translation verticale $w$, une rotation $\theta_{0}$ de l'extrémité droite de la buse située à gauche du nceud et une rotation $\theta_{d}$ de l'extrémité gauche de la buse située à droite du nœeud.

Le comportement du joint est supposé élastique, avec une rigidité de flexion $R^{\prime} j$ (de dimension F.L). On peut donc écrire des relations supplémentaires entre les rotations aux ncuds et les moments aux extrémités des buses:

$$
\begin{aligned}
& M_{d}=R^{\prime} j \cdot\left(\theta_{d}-\theta_{g}\right) \\
& M_{d}=R^{\prime} j \cdot\left(\theta_{a}-\theta_{d}\right)
\end{aligned}
$$

\section{3}

\section{Résolution}

Après l'assemblage de la matrice de rigidité $\mathrm{K}$ et du vecteur de charge $(F)$ de la structure, le système d'équation $(\mathrm{F})=\mathrm{K}$. $\mid \mathrm{U})$ est résolu (par la méthode de Gauss) pour obtenir le vecteur des déplacements nodaux $\{\mathrm{U}\}$, On obtient donc pour chaque nœud, le déplacement vertical $w$, et les rotations gauche et droite $\theta_{g}$ et $\theta_{\mathrm{d}}$.

En phase de post-traitement, on calcule des grandeurs qui permettent de qualifier certaines performances représentatives des tronçons de réseau:

- le vecteur $\left(\mathrm{F}^{e}\right)$ des efforts intérieurs (effort tranchant et moment de flexion) aux extrémités des poutres est calculé en effectuant le produit matriciel de la matrice de rigidité élémentaire $\mathrm{K}^{\circ}$ et du vecteur des déplacements nodaux $\left\{\delta^{c}\right\}$. On pourra en déduire les contraintes longitudinales susceptibles de provoquer la fissuration ou la rupture des buses;

- l'amplitude de l'ouverture des joints $\left(\theta_{i}-\theta_{d}\right)$ conditionne les fuites éventuelles ou les infiltrations dans le réseau;

- les pentes moyennes des buses sont calculées à partir des déplacements verticaux des joints.

Elles indiquent l'intensité de la contre-pente, qui peut s'opposer à l'écoulement des effluents.

\section{6}

\section{Modélisation du comportement d'un tronçon et mise en évidence de l'interaction}

On considère un tronçon de conduites de $120 \mathrm{~m}$ de longueur soumis à une charge continue et constante de $100 \mathrm{kN} / \mathrm{m}$ et dont les caractéristiques de référence 
sont définies au tableau IV. On entend par caractéristiques de référence, les données géométriques, matérielles de la conduite et du sol utilisées dans la suite de l'article lorsque aucune indication contraire n'est effectuée. L'échelle de l'étude est celle du tronçon de conduites.

La distribution choisie pour le coefficient de réaction du sol k est celle d'une distribution log-normale, étant donné que $\mathrm{k}$ a nécessairement une valeur positive.

Le sol est subdivisé en 480 zones (soit des zones de $25 \mathrm{~cm}$ de longueur) et chaque buse de la conduite est discrétisée en 12 éléments finis P2. Des études particulières ont permis de vérifier, sur des simulations déterministes, que le résultat était indépendant de la discrétisation choisie en zones, sous réserve d'un maillage suffisamment fin. En fait, l'exigence de finesse est relative à la précision nécessaire au calcul par éléments finis, mais aussi à la modélisation de la variabilité spatiale: la taille de la zone élémentaire doit être significativement plus petite que la longueur de corrélation pour que les variations à faible distance soient correctement reproduites. Les conditions aux limites constituées par la présence des regards, aux extrémités du tronçon de conduite, sont considérées dans cette étude comme libres.

Le coefficient de variation correspond à :

$$
\text { C.V. }=\frac{\sigma}{\mathrm{k}}
$$

Il est difficile de donner des valeurs numériques des raideurs de joints reposant sur des mesures, car les joints sont de géométries complexes, très diverses et leur raideur a fait l'objet de peu d'analyses. Nous avons choisi de contourner cette difficulté en introduisant le paramètre $\mathrm{L}_{\text {fictivg }}$ : la raideur du joint est égale à celle d'un tronçon (fictif) de buse, de mêmes caractéristiques géométriques et matérielles que la conduite voisine, et de longueur égale à $\mathrm{L}_{\text {fítive. }}$ Ainsi, la raideur du joint peut varier de zéro (quand $L_{\text {fictive }}$ tend vers l'infini) à l'infini (quand $L_{\text {fictive }}$ tend vers zéro), La longueur de cet élément est fictive dans la mesure où cette raideur est concentrée en un point matériel, le nceud, où l'on applique la relation (15). Nous pouvons ainsi procéder à des analyses de sensibilité, qui justifieront (ou pas) de procéder à des études plus fines des raideurs de joints réels.

Trois familles de facteurs gouvernent le comportement du système sol-conduites: la nature du sol, le matériau et la géométrie des conduites, la nature des joints de liaison.

A la figure 5 sont présentés respectivement les variations longitudinales du coefficient de réaction du sol, du moment fléchissant et du déplacement vertical pour des terrains présentant les mêmes caractéristiques statistiques mais des longueurs de corrélation différentes $l_{1}=3$ et $30 \mathrm{~m}$, soit de 1 à 10 fois la longueur d'un élément de conduite). Le tracé est restreint à une zone de $20 \mathrm{~m}$ de long, éloignée des limites du tronçon.
Le signal $k(x)$ est beaucoup plus agité (de fluctuation rapide) pour une faible longueur de corrélation $\left(1_{e c} / L_{\text {puse }}\right.$ $=1$ ) que pour $\left(l_{1} / L_{\text {mut }}=10\right)$. Dans ce dernier cas, les fluctuations sont plus lentes, le signal est plus régulier à l'échelle considérée.

Les signaux $w(x)$ sont beaucoup plus réguliers que ceux de $\mathrm{k}(\mathrm{x})$. En fait, la conduite agit comme un « filtre» de la variabilité, puisqu'une variation longitudinale de $w(x)$, qui résulte de la variation de raideur $k(x)$ doit aussi être compatible avec la rigidité de la buse. Le résultat est intermédiaire entre deux cas extrêmes:

- buse infiniment souple, situation dans laquelle w(x) est inversement proportionnel à $\mathrm{k}(\mathrm{x})$, la raideur en un point conditionnant totalement le déplacement au même point;

- buse et joints infiniment rigides, situation dans laquelle les déplacements sont constants, et ne sont donc plus liés à la raideur locale, l'ensemble du tronçon se comportant comme un corps rigide.

Dans la situation réelle, les déplacements w(x) dépendent donc des $\mathrm{k}(\mathrm{x})$ dans le voisinage de ce point et de la latitude que permet la souplesse du système pour des variations locales de $w(x)$. Ainsi, si la figure $5 d$ confirme que les déplacements les plus faibles sont relevés dans la zone de plus forte raideur $k$ (autour de $90 \mathrm{~m}$ ), la figure $5 \mathrm{c}$ montre qu'une variation très locale ne conduit pas nécessairement à de forts déplacements au même endroit, incompatibles avec la rigidité dư système.

Le même type de réponse est manifeste sur les courbes de moment fléchissant (Fig. 5e et 5f). Le moment fléchissant est proportionnel à la courbure locale, donc régi par la rigidité des buses et des joints. Les fluctuations à grande échelle restent possibles, mais celles à courte échelle sont fortement atténuées. Les fonctions $\mathrm{M}(\mathrm{x})$ montrent des changements de signe, correspondant à des changements de courbure, mais l'amplitude des variations (entre $-60 \mathrm{kN}$.m et $44 \mathrm{kN} \cdot \mathrm{m}$ pour $\mathrm{I}_{\mathrm{ec}} / \mathrm{L}_{\text {buse }}=1$ et entre $-6 \mathrm{kN}$.m et $93 \mathrm{kN} . \mathrm{m}$ pour $I_{\mathrm{e}} / \mathrm{L}_{\text {buse }}=10$ ) n'est pas significative à cette échelle. Notons seulement que les valeurs relevées sont conséquentes (plusieurs dizaines de m.kN) alors que négliger les effets de la variabilité longitudinale conduit naturellement à des moments nuls.

Une analyse appropriée de l'interaction doit reposer sur un traitement statistique des résultats, aussi bien à l'échelle du tronçon qu'à celle d'une population de tronçons. Dans un premier temps, nous allons étudier les corrélations entre grandeurs locales (rigidité, déplacements, moments fléchissants) dans un tronçon, quand varie la longueur de corrélation (\$7) ou la rigidité des joints (\$8). Nous verrons ensuite comment les résultats peuvent être exploités de façon statistique, à l'échelle d'une population de tronçons, en estimant les risques de dépassement de valeurs critiques de certaines grandeurs, et comment ces risques varient en fonction de l'échelle de fluctuation du sol.

TABLEAUIV Caractérístiques de référence.

Characteristics of reference.

\begin{tabular}{|c|c|c|c|c|c|c|c|}
\hline & Sol & & \multicolumn{4}{|c|}{ Conduite } & Joint \\
\hline $\begin{array}{c}k \\
\left(\mathrm{MN} / \mathrm{m}^{2}\right)\end{array}$ & c.v. & $\begin{array}{l}\mathrm{I}_{\mathrm{ec}} \\
(\mathrm{m})\end{array}$ & $\underset{(\mathrm{m})}{\mathrm{d}}$ & $\begin{array}{c}\mathrm{e} \\
(\mathrm{m})\end{array}$ & $\begin{array}{c}E c \\
(\mathrm{MPa})\end{array}$ & $\begin{array}{l}\mathrm{L}_{\text {buse }} \\
(\mathrm{m})\end{array}$ & $\begin{array}{l}\mathrm{L}_{\text {Tratie }} \\
(\mathrm{m})\end{array}$ \\
\hline 20 & 0,3 & 3 & 1.5 & 0,15 & 2. $10^{4}$ & 3 & 3 \\
\hline
\end{tabular}


a

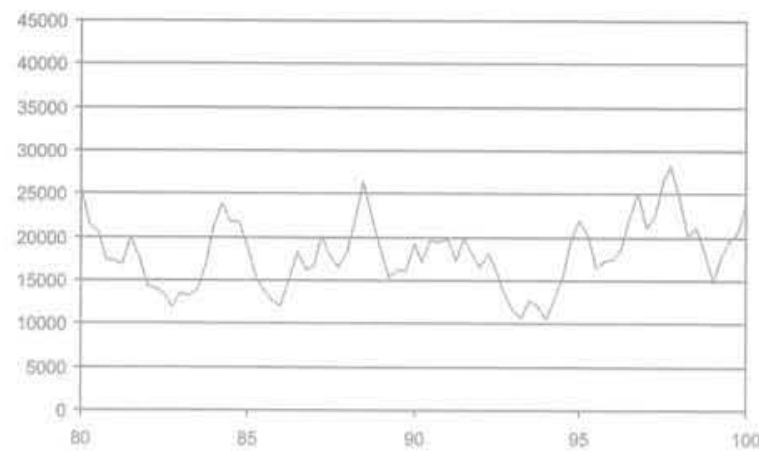

C

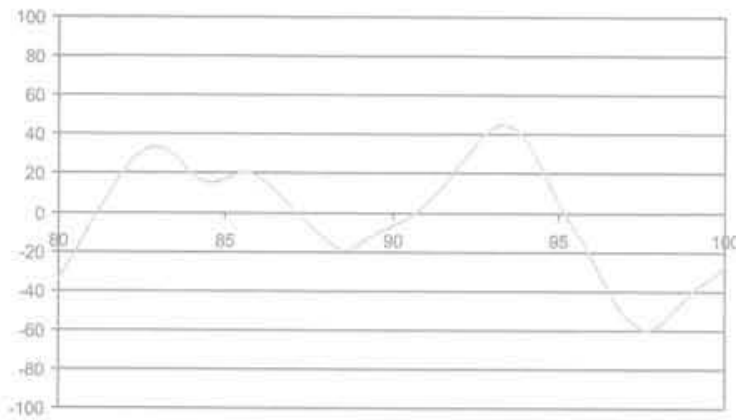

$\mathrm{e}$

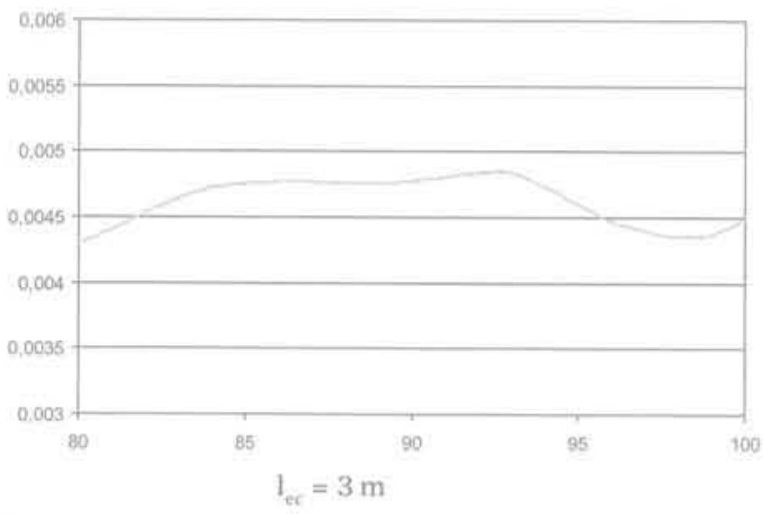

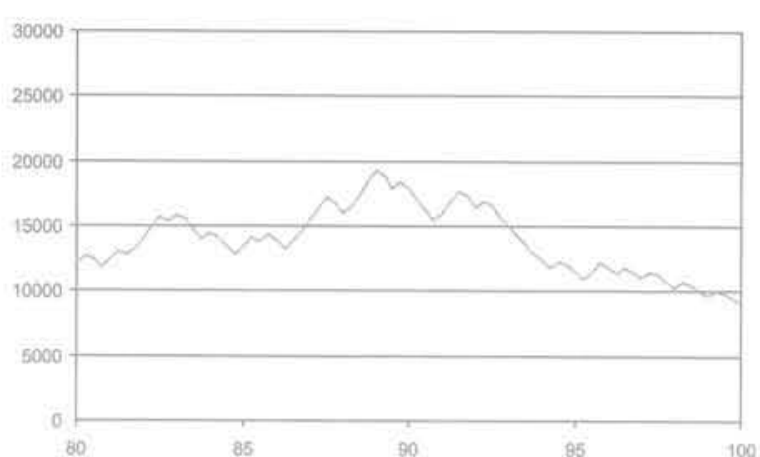

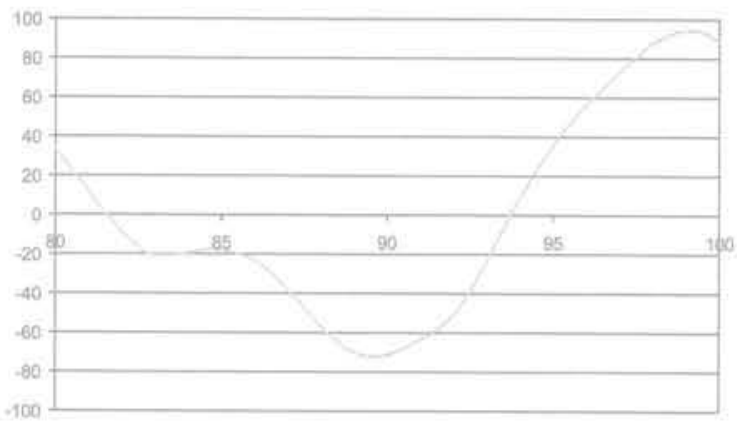

d

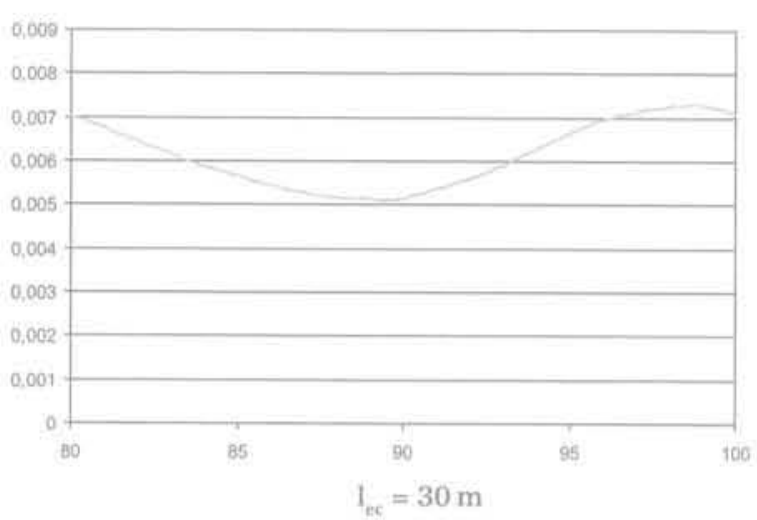

FG. $5 \mathrm{k}\left(\mathrm{en} \mathrm{kN} / \mathrm{m}^{3}\right), \mathrm{M}(\mathrm{en} \mathrm{kN} . \mathrm{m})$ et $\mathrm{w}(\mathrm{en} \mathrm{m})$ pour un tronçon de conduite $(80 \mathrm{a} 100 \mathrm{~m})$ correspondant à deux longueurs de fluctuation ( 3 et $30 \mathrm{~m}$ ).

$\mathrm{k}$ (in $\mathrm{kN} / \mathrm{m} 3$ ), $\mathrm{M}$ (in $\mathrm{kN} . \mathrm{m}$ ) and $w$ (in $\mathrm{m}$ ) for a section pipe ( 80 to $100 \mathrm{~m}$ ) corresponding to two lentghs of correlation (3 and $30 \mathrm{~m})$.

\section{7}

\section{Analyse du rôle de la longueur de corrélation \\ sur l'interaction sol-conduite}

Afin d'analyser l'effet de la longueur de corrélation sur la réponse (moment fléchissant, tassement) de la conduite, il est intéressant de quantifier les corrélations statistiques entre le vecteur module de réaction $\mathrm{k}(\mathrm{x})$ et les vecteurs, résultant des simulations numériques, déplacement vertical de la conduite $(w(x))$ et moment de flexion $(\mathrm{M}(\mathrm{x}))$. Pour chaque tronçon simulé, on peut ainsi calculer les coefficients $R_{w v}(k(x), w(x))$ et $R_{M}(k(x)$, $M(x)$ ) puis étudier la variation de ces coefficients pour des valeurs différentes $I_{\text {ec }}$. La figure 6 présente cette évolution. Chaque point est tracé à partir de la corrélation établie sur un ensemble de trois réalisations (soit 1440 valeurs de $\mathrm{x}$ ).

Les courbes des figures $6 \mathrm{a}$ et $6 \mathrm{~b}$ présentent des allures différentes: variation monotone avec un $R_{w}$ décroissant quand $l_{e c}$ croît pour les déplacements
(Fig. 6b), non monotone avec une plage de 1 conduisant à une corrélation maximale (en valeur absolue) pour les moments fléchissants (Fig. 6a).

Pour la réponse en termes de tassement, la corrélation tend ver's zéro si l tend vers zéro. Dans ce cas, les buses reposent sur un milieu extrèmement désordonné. Du fait de leur (relative) rigidité, le déplacement de la buse ne dépend plus de la valeur locale de $k(x)$ mais de sa moyenne sur une zone étendue: la corrélation entre déplacement et module local est donc très faible. L'autre cas limite est celui de I tendant vers l'infini. Le sol varie alors à très grande échelle, mais demeure quasiment uniforme à l'échelle d'une buse (variation spatiale tres «lente $)$ ). La relative souplesse de la buse permet au système de se déformer suffisamment pour qu'en chaque point, le déplacement soit inversement proportionnel à la valeur moyenne du module dans la zone considérée, elle-même très proche de la valeur locale du module. Les réponses observées peuvent aussi être analysées à la lumière des expressions ( $3 \mathrm{~b}$ ) et (5): dans le premier cas, les coefficients de réaction du sol de deux zones voisines le numérateur tend vers zéro, tandis que dans le second cas c'est le dénominateur qui tend vers l'infini. Pour toute valeur 


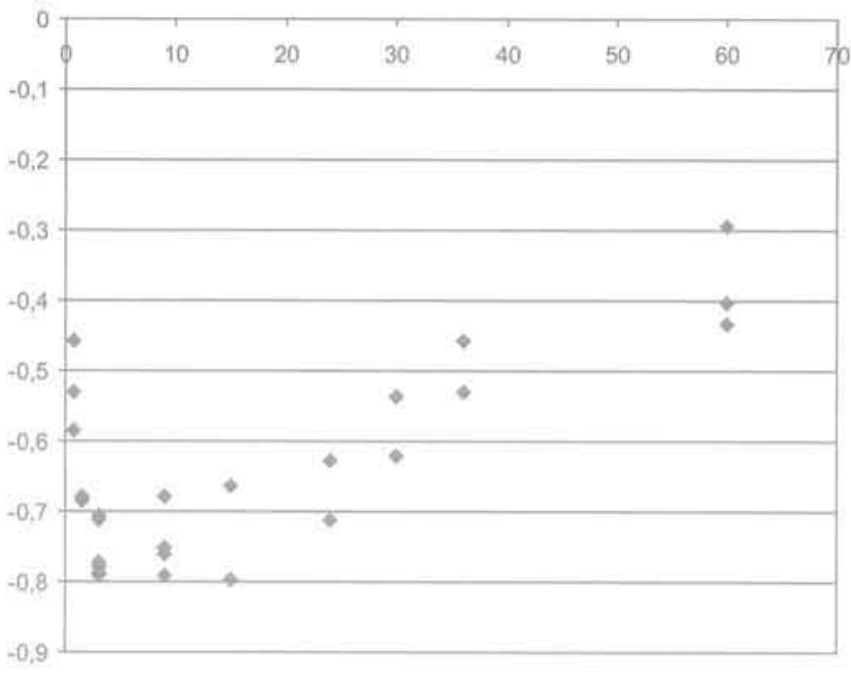

a

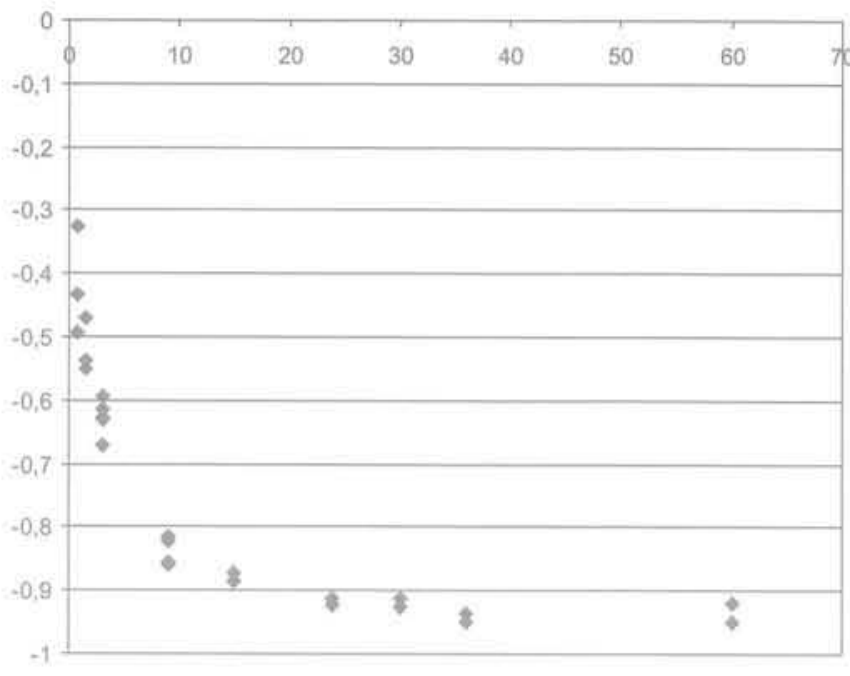

b

FG.6 Coefficients de corrélation en fonction de la longueur de corrélation $I_{e c}$; a) $\left.R_{M}-I_{e c}(e n m) ; b\right) R_{w}-I_{e f}(e n m)$. Coefficients of correlation function of the length of correlation $\left.\left.I_{n r}: a\right) R_{m}-I_{e c}(i n m) ; b\right) R_{w}-I_{n s}$ (in m).

finie de I la corrélation est intermédiaire, avec une transition progressive de la corrélation nulle vers la corrélation parfaite. La vitesse avec laquelle on converge vers une corrélation voisine de 1 dêpend de la "souplesse relative (ou «rigidité relativen de la buse), c'est-à-dire de sa capacité à s'accommoder des contrastes locaux de raideur du sol, ou à les filtrer. Pour les données de référence, le coefficient de corrélation atteint $-0,9$ pour $1 / L_{\text {buse }}$ voisin de 20 , soit des échelles de fluctuations de lordre de 60 mètres.

La figure 6 a montre que la relation entre module et moment est plus complexe. En fait, les deux cas extrêmes $\left(l_{\mathrm{ec}} \rightarrow 0\right.$ et $I_{\mathrm{ec}} \rightarrow$ infini) peuvent être comparés : dans les deux situations, le déplacement est quasiment uniforme sous une buse, soit parce que la variation de module, très rapide est filtrée, soit parce que, très lente, elle ne provoque pas de flexion (le moment fléchissant est proportionnel à la dérivée seconde de $w(x))$. Dans ces deux situations extrêmes, le coefficient de corrélation tend donc vers zéro. La situation la plus complexe est la situation intermédiaire, quand les fluctuations locales des propriétés du sol ne sont qu'incomplètement filtrées par les conduites et provoquent des déplacements $w(x)$ variables et des moments fléchissants $\mathrm{M}(\mathrm{x})$ significatifs. Pour une rigidité relative donnée (ici célle de la configuration de référence), il existe donc une valeur optimale de ! $/ \mathrm{L}_{\text {uuse }}$ à laquelle correspond une corrélation maximale (en valeur absolue) : c'est quand le rapport $1 / L_{\text {huge }}$ est compris entre 3 et $12 \mathrm{~m}$ ( 1 à 4 fois la longueur de la buse) que les moments fléchissants (positifs et négatifs) sont les plus élevés. Cette plage de valeurs dépend des rigidités relatives conduite/joint/sol, d'une façon qu'il conviendra d'analyser plus précisément.

\section{8}

\section{Effet des joints de liaison}

La contribution de la raideur du joint de liaison entre deux buses peut être perçue à la figure 7 où est présenté le coefficient de corrélation en fonction de la longueur $\mathrm{L}_{\text {intive }}$ (rappelons que ce paramètre augmente avec la souplesse du joint: $L_{\text {fictive }}=0$ pour un joint infi- niment rigide et $\mathrm{L}_{\text {fictive }}$ tend vers l'infini pour une articulation). Un joint tres rigide $\left(\mathrm{L}_{\text {fictive }}<1 \mathrm{~m}\right.$ ) assure la transmission des efforts et des moments fléchissants entre buses, ce que ne fait pas un joint souple. Le joint, selon sa raideur, modifie donc la structure de corrélations entre l'entrée $k(x)$ et les sorties $w(x)$ et $M(x)$.

La variation des coefficients de corrélation, en dépit d'un bruit statistique important, est manifeste dans la plage de variation considérée. Par rapport à la configuration de référence (avec $\mathrm{L}_{\text {buse }}=3 \mathrm{~m}, 1_{\mathrm{cc}}=3 \mathrm{~m}, \mathrm{~L}_{\text {fictivo }}=$ $3 \mathrm{~m}$ ), les effets majeurs sont ceux d'une amélioration de

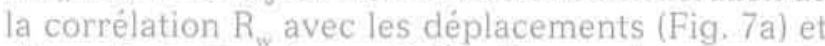
d'une diminution de corrélation $R_{M}$ avec les moments (Fig, 7b) pour des joints plus souples.

En pratique, la réponse du tronçon dépend donc aussi de la raideur des joints: une conduite constituée d'éléments rigides peut avoir un comportement longitudinal d'ensemble flexible si les joints sont flexibles (Fig. 7b)

\section{Analyse statistique}

Après cette étude analytique de la sensibilité aux différents paramètres d'influence, nous souhaitons traduire les résultats des simulations en termes de risques pour les conduites. Le dimensionnement des ouvrages de génie civil, dans le cadre des codes aux états limites, repose sur le concept fondamental de probabilité acceptée de défaillance. Le tronçon de réseau placé dans un sol hétérogène subit des déformations, déplacements et contraintes auxquelles on peut faire correspondre des variables de calcul et des états limites (ultimes ou structurels, de service ou fonctionnels), que l'on souhaite voir satisfaits avec une fiabilité suffisante.

Répéter les simulations numériques pour une configuration figée permet, en évaluant les déplacements et moments fléchissants extrêmes pour chaque simulation, de construire les lois de distributions statistiques (ou les lois de répartition) des sollicitations subies par le tronçon. On peut donc en déduire, dans un second temps, les valeurs correspondant à certains fractiles. 


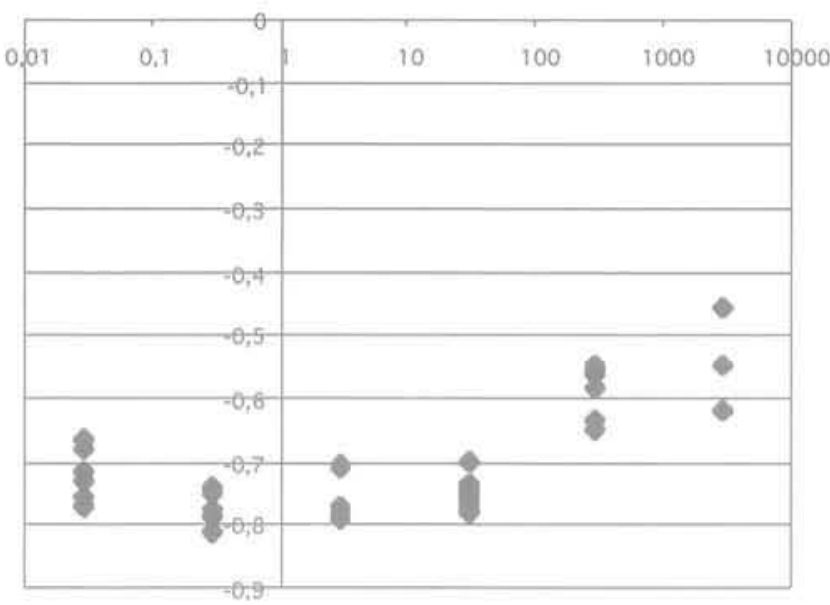

a

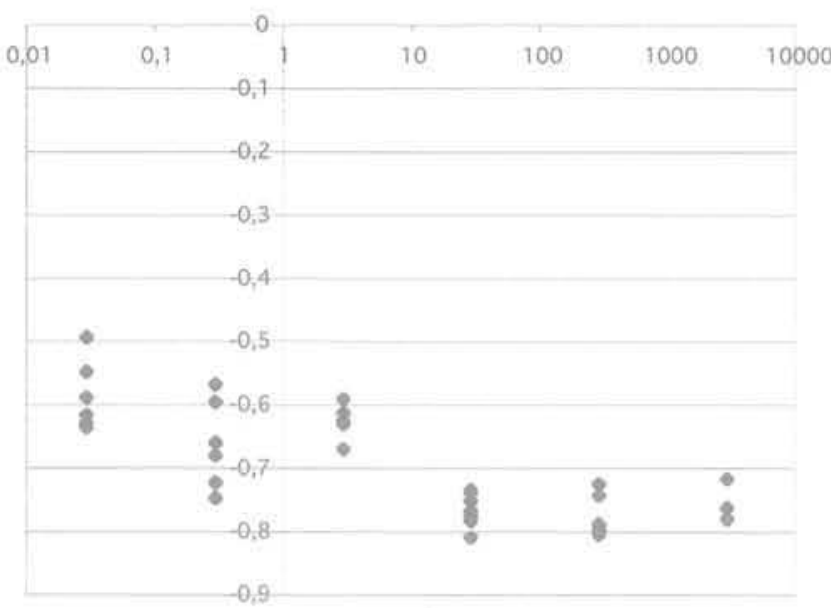

b

FIG.7 Coefficients de corrélation en fonction de la longueur fictive $\left.\left.I_{\text {nilive }}: a\right) R_{M}-I_{\text {fictive }}(e n m) ; b\right) R_{w}-I_{\text {native }}(e n m)$. Coefficients of correlation function of the fictive length $L_{\text {fiare }}:$ a) $R_{M}-L_{\text {fictive }}$ (in $\left.m\right) ; b$ ) $R_{w}-L_{\text {fistive }}$ (in $m$ ).

On appellera par exemple M95 la valeur du moment fléchissant qui (en valeur absolue), n'est atteint ou dépassé (à une abscisse quelconque) que dans 5 tronçons sur 100 (soit un risque de $5 \%$ ).

La méthode de Monte-Carlo est utilisée pour analyser la réponse statistique d'un tronçon de conduites. La taille de l'échantillonnage est de 1000 réalisations, taille satisfaisante pour un calcul linéaire. Pour chaque réalisation les réponses suivantes sont répertoriées:

- le moment fléchissant maximal $\mathrm{M}_{\max }$ appliqué le long de la conduite;

- la contre-pente maximale $\mathrm{CP}_{\max }(\mathrm{en} \%)$ définie par:

$$
\mathrm{CP}_{\max }=\left|\frac{{ }^{w} \mathrm{~d}^{-w} \mathrm{~g}}{\mathrm{~L}_{\text {buse }}}\right| \quad \mathrm{i}=1 \text {, nombre de buses }
$$

$\mathrm{w}_{\mathrm{d}}$ et $\mathrm{w}_{\mathrm{g}}$ correspondent aux flèches respectivement des extrémités droite et gauche d'une buse.

Ces deux réponses caractéristiques revêtent une importance particulière, La première permet de prendre en compte l'aspect mécanique du problème, puisqu'elle définit les contraintes maximales de flexion dans la conduite. L'aspect hydraulique est quant à lui repris par la seconde car les conduites d'assainissement ayant un écoulement gravitaire, une contre-pente importante constitue un obstacle à l'écoulement naturel et provoque des dysfonctionnements hydrauliques (ralentissement des effluents, ensablement, bouchage...).

Les figures 8 et 9 montrent les fonctions de répartition $\mathrm{F}$ obtenues pour trois valeurs de la longueur de corrélation ( $1 / L_{\text {buse }}=0,4,1$ et 5 ). Attachons-nous d'abord aux ordres de grandeur: pour la configuration de référence, un moment fléchissant de $100 \mathrm{kN} . \mathrm{m}$ correspond à une contrainte de traction sur la fibre la plus tendue de l'ordre de 0,5 MPa, susceptible de fissurer la conduite: ce moment fléchissant n'est pas pris en compte dans le dimensionnement habituel du réseau, puisque la variation longitudinale n'est pas considérée dans les calculs. Ajoutons que ces valeurs peuvent être beaucoup plus élevées pour un sol moins raide (la valeur du module de réaction de la configuration de référence est une valeur moyenne). En outre, la dispersion des réponses est importante et les risques d'atteindre des valeurs sensiblement plus élevées que la moyenne non négligeable.
L'effet de la longueur de fluctuation est particulier: $\mathrm{c}^{\prime}$ est pour $\mathrm{l}_{\mathrm{e}} / \mathrm{L}_{\text {buse }}=1$ que les moments fléchissants sont les plus élevés (ce qui confirme les résultats de la figure 6b). Considérer la structure de corrélation spatiale apparait donc essentiel, puisque c'est pour des valeurs intermédiaires de la longueur de fluctuation (de l'ordre de la longueur de la buse) que les contraintes générées sont les plus défavorables.

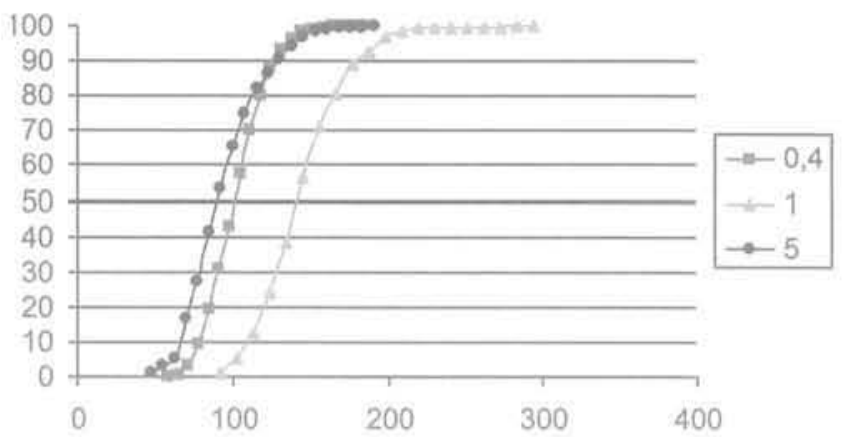

FG. 8 Fonction de répartition du Moment maximal $M_{\max }$ (en $\left.\mathrm{kN} . \mathrm{m}\right)$ pour 3 valeurs de $1_{\text {ej }} / L_{\text {buse }}(0,4,1$ et 5$)$.

Cumulative distribution function of the maximal bending Moment $M_{\text {max }}$ (in $\mathrm{kN}, \mathrm{m}$ ) for 3 values of $I_{0} / h_{\text {noss }}(0,4,1$ and 5$)$.

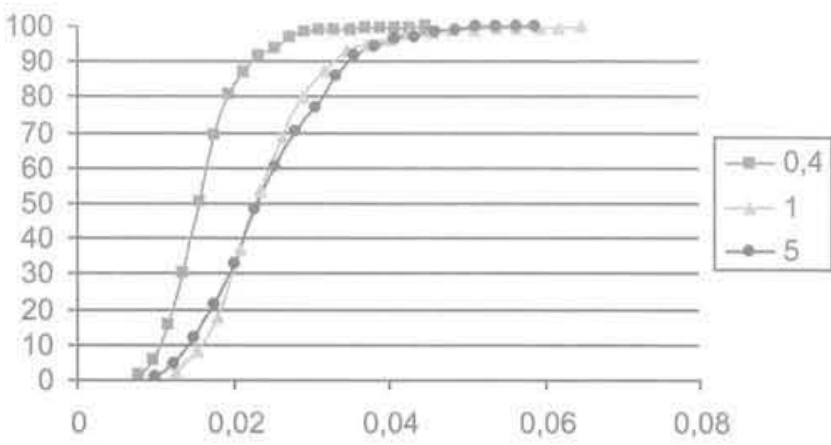

FIG. 9 Fonction de répartition de la contre-pente maximale $C P_{\max }$ pour 3 valeurs de $1_{\mathrm{ec}} / \mathrm{L}_{\text {buse }}$ $(0,4,1$ and 5$)$.

Cumulative distribution function of the maximal Counterslope $\mathrm{CP}_{\max }$ for 3 values of $\mathrm{I}_{2} / \mathrm{L}_{\text {base }}(0.4,1$ and 5). 
Des constatations similaires peuvent être faites en ce qui concerne la contre-pente (Fig 9), à la différence près que les valeurs $I_{\mathrm{ec}} / \mathrm{L}_{\text {buse }}=1 \mathrm{et} \mathrm{I}_{\mathrm{ec}} / \mathrm{L}_{\text {buse }}=5$ fournissent des résultats aussi défavorables (ce n'est qu'avec des rapports plus faibles, ici 0,4, que les contre-pentes sont atténuées, la variabilité longitudinale étant filtrée). Nous confirmons que la longueur de corrélation la plus défavorable n'est pas la même selon le paramètre étudié, comme cela a été montré dans le cas des tassements d'ouvrages (Breysse, 2001).

On peut analyser les risques de façon plus synthétique à partir des fractiles caractéristiques (au sens de "valeur caractéristique des actions $»$ dans les textes Eurocodes], on définit ainsi:

M95 tel que $\mathrm{p}(\mathrm{M}<\mathrm{M} 95)=0,95$

et CP95 tel que $p\left(\mathrm{CP}_{\max }<\mathrm{CP} 95\right)=0,95$

où $\mathrm{M}_{\text {max }}=\sup _{\mathrm{x}}(\mathrm{M}(\mathrm{x}))$ et $\mathrm{CP}_{\max }=\sup _{\mathrm{x}}(\mathrm{CP}(\mathrm{x}))$.

La figure 10 synthétise l'influence combinée des paramètres $k$ (module de réaction) et $\mathrm{l}_{\mathrm{e}} / \mathrm{L}_{\text {buse }}$ sur la valeur caractéristique M95 (valeur qui est statistiquement dépassée pour $5 \%$ des tronçons). La figure $10 \mathrm{a}$ présente les résultats en trois dimensions, les figures $10 \mathrm{~b}$ et $10 \mathrm{c}$ illustrant les traces de la même surface dans les plans verticaux: influence de $\mathrm{k}$ pour la figure $10 \mathrm{~b}$, influence de $1 / L_{\text {burs }}$ pour la figure $10 \mathrm{c}$. La figure 11 est construite selon le même principe pour la contrepente CP95.

L'influence du module de réaction est la plus simple, les valeurs de M95 et CP95 étant approximativement inversement proportionnelles à $k$, quelle que soit la valeur de $\mathrm{l}_{\mathrm{ec}} / \mathrm{L}_{\text {buse. }}$. A l'inverse, pour toute valeur de $\mathrm{k}$, les surfaces (Fig. 10a et 11) présentent des maxima pour une plage de valeur particulière du rapport $1 / L_{\text {buse }}$ illustrant le caractère fortement non linéaire de l'interaction entre les données $\mathrm{k}-1_{\text {er }}$ et les sorties M95 et CP95. Les valeurs les plus défavorables (moments maximaux ou contre-pentes maximales) sont atteintes pour des longueurs de fluctuation $I_{\text {ei }}$ de l'ordre de 1 à 2 fois la longueur de buse $L_{\text {buse }}$ pour M95 et de l'ordre de 2 à 3 fois cette longueur pour CP95.

L'effet de la variabilité du module de réaction peut aussi être appréhendé. Les figures $12 \mathrm{a}$ et $12 \mathrm{~b}$ présentent les résultats obtenus pour M95 et CP95 quand on fait varier les valeurs moyennes de $k$ (entre 10 et $100 \mathrm{MN} / \mathrm{m}^{3}$ ) et leur coefficient de variation (entre 0 , cas homogène, et $80 \%$ ). Les moments et contrepentes croissent de façon proportionnelle à la dispersion du paramètre $k$. Ces figures confirment qu'il est préférable, en termes de comportement mécanique longitudinal, d'avoir affaire à un sol ordinaire mais homogène (par exemple $\mathrm{k}=30 \mathrm{MN} / \mathrm{m}^{3}, \mathrm{c} . \mathrm{v} .=15 \%$ ) qu'à un sol plus compact en moyenne mais avec un coefficient de variation élevé (par exemple $\mathrm{k}=80 \mathrm{MN} / \mathrm{m}^{3}, \mathrm{c}, \mathrm{v} .=60 \%$ ).

\section{0}

\section{Conclusion}

Un modèle simple permettant de modéliser l'interaction sol-conduite en tenant compte de la variabilité longitudinale du sol a été développé. Il est appliqué à l'analyse du comportement d'un tronçon de conduite d'assainissement. Le sol et la conduite sont discrétisés à des échelles qui permettent de représenter finement la variation spatiale des rigidités.

Une analyse probabiliste (méthode de Monte-Carlo) permet, via une étude paramétrique, de recenser et d'étudier les principaux facteurs gouvernant le com-
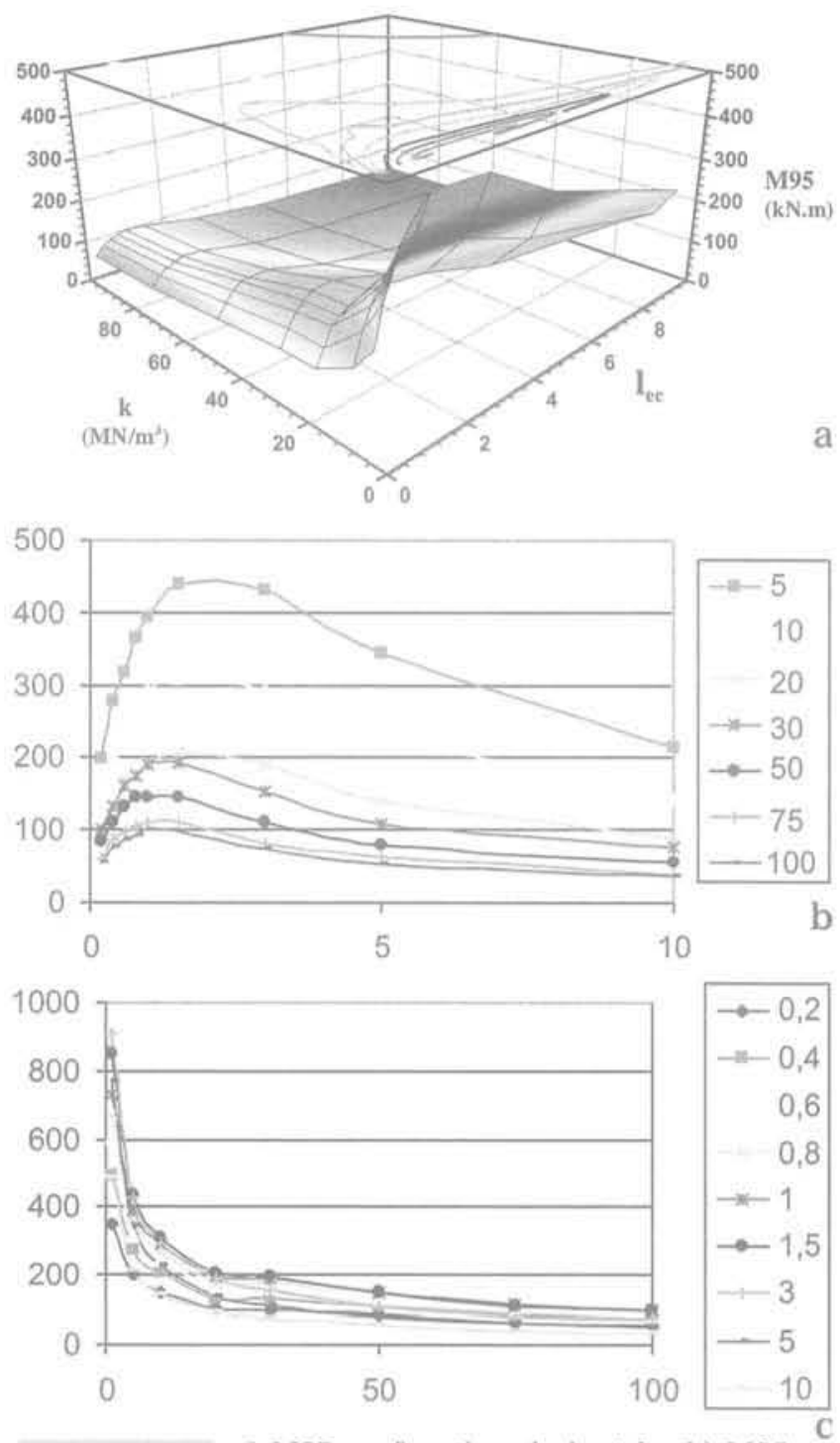

FIG. 10 a) M95 en fonction de $k$ et $1_{\text {e }}$; b) M95 en fonction de $1 / 1$ ( $/$ variant de 1 à $\left.120 \mathrm{MN} / \mathrm{m}^{3}\right)$; c) $\mathrm{M}^{2} 5$ en fonction de $\mathrm{k}\left(1_{\mathrm{eg}}\right.$ $\Lambda_{\text {bus }}$ variant de 0,2 à 10 ).

a) M95 function of $\mathrm{k}$ and 1 ; b) $\mathrm{M} 95$ function of 1. $/ \mathrm{L}_{\text {time }}\left(\mathrm{k}\right.$ varies with 1 to $\left.120 \mathrm{MN} / \mathrm{m}^{3}\right)$; c) $\mathrm{M} 95$ function of $\mathrm{k}\left(0_{\mathrm{e}} /\right.$ isuse varies with 0.2 to 10$)$.

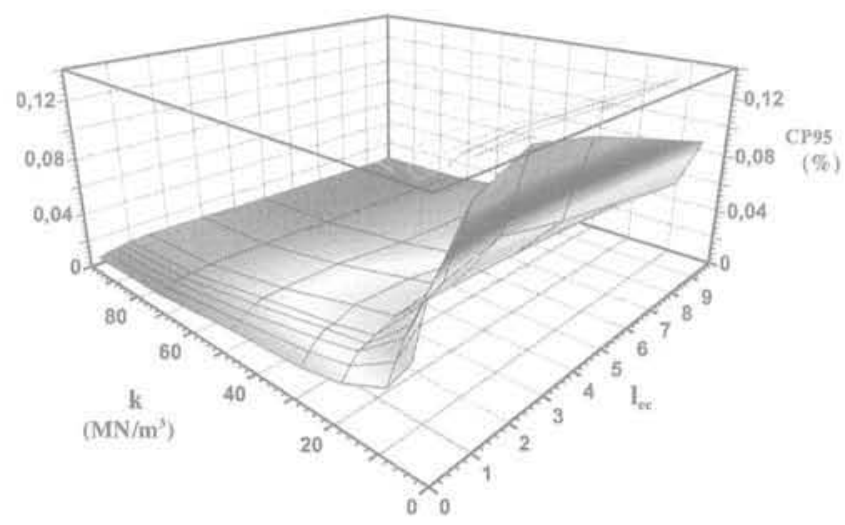

FIG. 11 CP95 en fonction de $\mathrm{k}$ et $\mathrm{l}_{\mathrm{e}}$ CP95 function of $\mathrm{k}$ and $\mathrm{I}_{\mathrm{e}}$.

portement longitudinal d'un tronçon de canalisations. On montre que les variables dominantes sont:

- la longueur spatiale de corrélation du sol; 

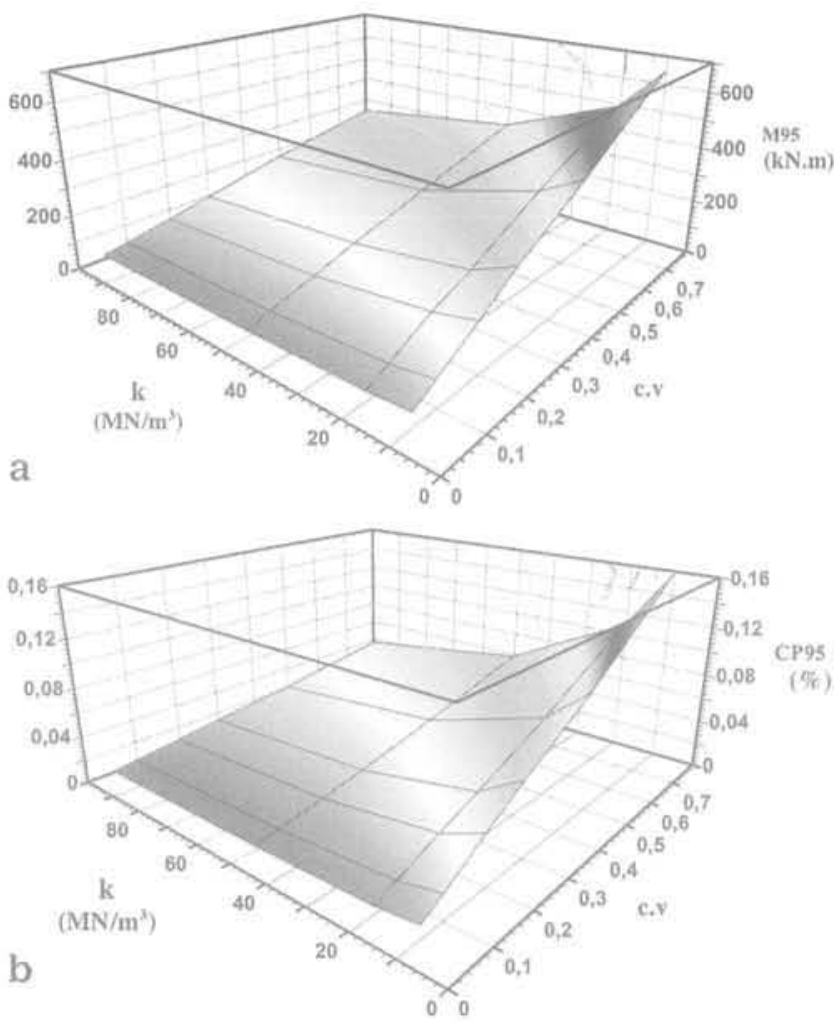

FIG, 12. a) : M95 en fonction de $k$ et de c.v. M95 function of $k$ and $c . v$.

b) : CP95 en fonction de k et de c.v. CP95 function of $k$ and $c . v$.
- le type de joint utilisé qui se manifeste par sa rigidité relativement à celle de la conduite;

- le type de matériau ainsi que les dimensions de la conduite.

La complexité de l'interaction sol-conduite a été analysée au travers des rapports entre les rigidités respectives qui conditionnent le comportement du système (rigidité du sol, des buses et des joints). On a montré que les effets les plus défavorables sont obtenus pour des valeurs particulières de la longueur de fluctuation des propriétés de sol, qui sont approximativement de l'ordre de grandeur de la dimension des éléments de conduite. Des échelles de fluctuation plus faibles ou plus fortes produisent des effets atténués, tendant vers l'homogénéité du système dans des cas extrêmes.

Le recours à des simulations de Monte-Carlo permet de quantifier les risques de dépasser certaines valeurs caractéristiques de déplacement ou de contrainte. Outre l'intérêt qu'il constitue pour mieux appréhender les phénomènes complexes d'interaction, un tel outil numérique constitue donc une base essentielle pour une prise en compte rationnelle des conséquences de la variabilité longitudinale pour le dimensionnement des réseaux enterrés. Les perspectives de développement à court terme concernent l'addition de la dimension temporelle des mécanismes de dégradation du sol, par exemple, l'intégration dans les simulations de l'altération progressive des modules de réaction du fait de l'érosion progressive du remblai (érosion interne sous l'effet des exfiltrations dans les joints ou les fissures). Un tel outil permettra, en outre, d'établir des lois réalistes d'évolution des défauts autour des conduites, qui pourront nourrir les réflexions menées actuellement dans le cadre de projets nationaux (RERAU; Le Gauffre, 2002).

\section{Bibliographie}

Baecher G.B. Ingra T.S. - - Stochastic FEM in settlement predictions w. Journal of Geotechnical Engineering, ASCE, 107 (4). 1981, p. 449-463.

Benmansour A., Abdallah A., Masrouri F. Auvinet G. - "Analyse fiabiliste du comportement axial des conduites d'assainissement 1). Revue canadienne de géotechnique, n०34, 1997, p. 329-343

Bensafi M., Nedjar D., Elachachi S.M. Hamane M., Breysse D. - « Analysis of burjed pipes subjected to a dynamic solicitation with no deterministic approach 1 . European Conference on dynamics, EURODYN'2002, Munich, 2-5 September 2002, Balkema.

Biot A.M. - Bending of an infinite beam on an elastic foundation 1). J. Applied Mechanics, ASME, vol. 4., 1937, A1-A7.

Breysse D., Boissier D. - "Lévaluation et la maintenance des réseaux d'assainissement 1 . Application des notions de fiabilité à la gestion des ouvrages existants. ss la dir de C. Crémona, AFGC, Presses des ponts et chaussées, 2002, p. 139-170.

Breysse D, - a Les enjeux de la modélisation des terrains et des structures pour décrire au mieux l'interaction sol-ouvrage 3. 1ro conf. Intern. A. Caquot, Presses des ponts et chaussées, Paris, 2001
Brevsse D. Elachachi S.M. Boukhoulda H. - Modélisation des désordres dans les réseaux enterrés consécutifs à l'hétérogénéité des sols w. JNGG, Nancy, 8-10, octobre 2002 .

Bucher C.G. Shinozuka M. - « Structural response variability 11 w, Journal of Engineering Mechanics, ASCE, 114, 12, 1988 , p. 2035-2054

Carmeliet J., Hans $\mathrm{H}_{\text {, }}$ - « Probabilistic non local damage model for continua with random field propertvm. Journal of Engineering Mechanics, ASCE, 120, 10, 1994 , p. 2013-2027.

Cheng R.H. Wen Y.K. - Reliability of uncertain nonlinear trusses under random excitation. Journal of Engineering Mechanics, ASCE, 120, 4, 1994, p. 748757

Der Kiureghian A., Ke J.B. - "The stochastic finite element method in structural reliability n. Probabilistic Engineering Mechanics, 3 (2), 1988, p. 83-91.

Fenton G.A., Van Marcke E.H. "Simulation of random fields via local average subdivision ग. Journal of Engineering Mechanics, ASCE, vol. 116, n० 8, 1990

Fenton G.A. Paice G.M. Griffiths D.V Proceedings of Uncertainty'96. ASCE conference, Madison, Wisconsin, 1996.
Filonenko-Borodich MM - «Some approximate theories of elastic foundation n. Uchenvie Zapiski Moskovskogo Gosudarstvennogo Universiteta (in Russian) Mekhanica 1940, 46; p. 3-18.

Grundmann $\mathrm{H}$. Waubke $\mathrm{H}$ - $-\alpha \mathrm{Ar}$ approach to stochastic nonlinear soil structure interaction under dynamic loading n. Proceed. Icossar'93, vol. 1. G.1. Schueller, M. Shinozuka and J.T.P. Yao (eds), Balkema, 1993, p. 341. 348.

Hetenyi M.A. - a General solution for the bending of beams on an elastic foundation of arbitrary continuity 1 . Journal of Applied Physics, n²1, 1950, p, 55-58.

Horvarth J.S, - a Modulus of subgrade reaction: new perspective 1. Journal of Geotechnical Engineering. ASCE. vol. 109, 12, 1983

Horvath J.S. - a Beam-column-analogy model for soil-structure interaction anaJysis n. Journal of Geotechnical Engineering, ASCE, vol. 119, 2, 1993, p. 358-364.

Jaksa M.B. - "The influence of spatial variability on the geotechnical design properties of a stiff, overconsolidated clay ". PHD thesis, University of Adelaide, Australia, 1995. 
Kerr A.D. $-\propto$ Elastic and viscoelastic foundation models 3. Journal of Applied Mechanics, ASME, 31, 4, 1964, p. 491-498.

Kerr A.D. - "A study of a new foundation model s. Acta Mech., 1/2, 1965, p. 135-147.

Kouraoaka S. - «Pipe soil interactions analysis of field tests of buried PVC pipe Journal of Engineering Mechanics. ASCE. 119, 5, 1993, p. 938-954.

Le Gauffre P. Joannis C.. Gibello C.. Breysse D. - i Performance indicators and decision support for rehabilitation of sewer networks \&. Contribution of the French R\&D RERAU program. SOM 2002, Bradford, GB, 26-28 nov. 2002.

Liu W.K., Mani A., Belytschko T. - "Finite element methods in probabilistic mechanics 1. Probabilistic Engineering Mechanics, 2 (4), 1987, p. 201-213.

MELT - Fascicule 70, Ouvrages d'assainissement. Textes officiels, circulaire $n^{\circ} 92$ 42 du 1 juillet 1992.
Nedjar D., Bensafi M., S.M. Elachachi, Hamane M., Breysse D. - «Buried pipe response under seismic solicitation with soil-pipe interaction n. NUMGE Conference, Presses des ponts et chaussées. 2002, p. 1047-1053.

Okeagu B., Abdel-sayed G. - "Coefficient of soll reaction of buried flexible conduits n. Journal of Geotechnical Engineering, ASCE, $110,7,1984$.

Pasternak P. L. - $\alpha$ On a new method of analysis of an elastic foundation by means of two foundation constants u) (in Russian). Gosudarstvennoe Izdatelstvo Literaturi po Stroitelstvu Arkhitekture, Moscow, USSR, 1954.

Selvadurai A.P.S. - "Soil-pipeline interaction during ground movement is. Arctic 1985. Civil Engineering in the Arctic Offshore, F.L. Bennett \& J.L. Machemehl (eds), ASCE Speciality Conference, San Francisco, 1985, p. 763-773

Ting B.Y.. Mockry E.F. - - Beam on elastic foundation finite element \%. Journal of Structural Engineering, ASCE, 110 (10), 1984, p. 2324-2339

VanMarcke E. - Random fields : analysis and synthesis. MIT Press, Cambridge, Massachusetts London, England, 1983.

VanMarcke E., Grigoriu M. - «Stochastic finite element analysis of simple beams 1. Journal of Engineering Mechanics, ASCE, 109, 5, 1983

Vlassov V.L.. Leontiev N.H. - "Beams plates and shells on elastic foundations n. Fizmatgiz, Moscow, 1956.

Vesic A.S. - "Beams on elastic subgrade and the Winkler hypothesis n. Proceed. Sth Int. Conf. Soil Mech. and Foundations Eng., Paris, vol. 1, 1963, p. 845-850.

Winkler E. - Die Lehre von der Elasticitaet und Festigkeit. Prag. Dominicus, 1867.

Zhang J.. Ellingwood B. - «Effects of uncertain material properties on structural stability w. Journal of Structural Engineering, ASCE, 121 (4), 1995, p. 705-716, 